\title{
Detrimental effect of Hypericum perforatum on ovarian functions
}

\author{
(D) Buket Demirci1 , (D) Fadime Kahyaoğlu², (D) Tolga Atakul³, (D) Mustafa Yılmaz4, (D Yavuz Özoran² \\ 1Department of Medical Pharmacology, Adnan Menderes University Faculty of Medicine, Aydın, Turkey \\ 2Department of Pathology Laboratory Tecniques, Avrasya University Vocational School of Health Services, Trabzon, Turkey \\ 3Department of Obstetrics and Gynecology, Adnan Menderes University Faculty of Medicine, Aydın, Turkey \\ 4Department of Medical Biochemistry, Adnan Menderes University Faculty of Medicine, Aydın, Turkey
}

\section{Abstract}

Objective: Hypericum perforatum is widely used for depression and distress treatment as an over-the-counter plant at any age. This study investigated the safety of $H$. perforatum on ovarian function and infertility.

Material and Methods: $H$. perforatum was given to rats in two different dosages (100 and $300 \mathrm{mg} / \mathrm{kg} / \mathrm{day}$ ) with drinking water for four weeks. Half of the treatment groups were sacrificed at the end of the four-week intervention, the remainder was sacrificed after an additional fourweek waiting period to see if there was reversibility. At the end of the experiment, blood samples and both ovarian tissues were obtained under anesthesia with ketamine and xylazine $(50 \mathrm{mg} / \mathrm{kg}$ and $5 \mathrm{mg} / \mathrm{kg}$, respectively).

Results: Although primordial follicle numbers were not affected with a dose of $100 \mathrm{mg} / \mathrm{kg}$, they were significantly decreased (28.6\%) when the dose was tripled. Primary follicle numbers stayed the same, but secondary and tertiary follicles numbers were significantly dose-dependently decreased, and remained significantly low four weeks after the intervention. Anti-mullerian hormone (AMH) levels were not significantly different between the groups.

Conclusion: $H$. perforatum treatment did not change serum levels of AMH because the primary follicle number did not decrease. However, the other follicle counts decreased in a dose-dependent manner and full recovery was not regained after four weeks. The detrimental effect of $H$. perforatum on primordial follicles should be taken into consideration because any woman using $H$. perforatum could also experience ovarian failure. (J Turk Ger Gynecol Assoc 2019; 20: 65-9)

Keywords: Anti-mullerian hormone, ovarian capacity, rational drug treatment, rat, St. John's wort

Received: 26 March, 2018 Accepted: 10 May, 2018

\section{Introduction}

Although psychological distress by itself can cause psychosomatic infertility or decrease treatment success (1), the treatments of distress and depression with medicines and plants may also have a negative impact on ovarian function. There is a growing interest in plants and their derivatives, with an assumption that they are a safe method of treatment. Patients with infertility are increasingly using complementary and alternative medicines to support or replace medical fertility treatments (2). Extracts from the plant Hypericum perforatum (St John's wort) have become an appealing alternative therapy to prescription serotonin-modulating drugs and are widely available to women of childbearing age $(3,4)$.

There is little research on $H$. perforatum and its use in pregnancy, and its effect on fertility has not been established, even in European Medicines Agency guidelines (EMA) (5). Therefore, we evaluated impact of $H$. perforatum on ovarian tissue by counting follicles and assessing anti-mullerian hormone (AMH) levels. 


\section{Material and Methods}

\section{Chemicals and animals}

H. perforatum (St. John's Wort Herb Extract/SOLGAR, İstanbul, Turkey) was obtained from a local pharmacy store. Thirty-five 16-20-week-old female Wistar rats were obtained from the university and all experiments were performed according to the principles and guidelines of the University Animal Ethical Committee's approval (HADYEK 64583101/2016/3). The "Principles of laboratory animal care" (NIH publication no. 86-23, revised 1985) and specific national laws were followed throughout the study. On the study day, the rats were randomly assigned to five groups of seven animals each.

\section{Experimental design}

Control group: The rats in this group were allowed free access to tap water.

Low-H. perforatum group: The rats in this group were administered $100 \mathrm{mg} / \mathrm{kg} \mathrm{H}$. perforatum with drinking water for four weeks.

Low-H. perforatum waiting group: The rats in this group were administered $100 \mathrm{mg} / \mathrm{kg} \mathrm{H}$. perforatum with drinking water for four weeks, and then the rats received no medication and were sacrificed 4 weeks later.

High-H. perforatum group: The rats in this group were administered $300 \mathrm{mg} / \mathrm{kg} H$. perforatum with drinking water for four weeks.

High-H. perforatum group: The rats in this group were administered $300 \mathrm{mg} / \mathrm{kg} \mathrm{H}$. perforatum with drinking water for four weeks, and then the rats received no medication and were sacrificed 4 weeks later.

The rats were weighed every monday and the doses of $H$. perforatum were adjusted for every cage. At the end of the experiment, under the anesthesia with ketamine and xylazine (50 $\mathrm{mg} / \mathrm{kg}$ and $5 \mathrm{mg} / \mathrm{kg}$, respectively), blood samples were obtained by cardiac puncture, centrifuged at $3000 \mathrm{rpm} / 4^{\circ} \mathrm{C}$, and sera were stored at $-80{ }^{\circ} \mathrm{C}$ for AMH measurement. In addition, both ovaries were harvested and kept in 10\% formalin.

The suggested daily dosage of $H$. perforatum for humans is 900 mg (or $15 \mathrm{mg} / \mathrm{kg}$ per day for a $60 \mathrm{~kg}$ human being); Rayburn et al. (3), calculated the rodent dosage as $180 \mathrm{mg} / \mathrm{kg}$ per day in their study. Considering that study, we wanted to apply two different doses of $H$. perforatum treatment to have a better understanding of dose effect and determined doses of 100 $\mathrm{mg}$ and $300 \mathrm{mg} / \mathrm{kg}$ (low and high). Additionally, we sacrificed some rats after a waiting period to determine if there was any reversibility of effect.

\section{Follicle counting}

After routine tissue processing, the obtained samples were sliced in 5-micrometer thickness and evaluated under an optical microscope (Zeiss Primo star, Ankara, Turkey) with hematoxylin-eosin (H-E) staining. Oocyte classification was based on previous studies $(6,7)$.

Primordial follicle; follicles comprising a central oocyte and surrounding monolayer of flat squamous granulosa cells (Figure 1a-c).

Primary follicles; a central oocyte and surrounding monolayer of cubic granulosa cells or at least 3 cubic epithelial cells in monolayer granulosa cells (Figure 1d-g).

Secondary follicle (pre-antral follicle); follicles containing two or more layers of granulosa cells, without formed antrum follicles (Figure 1h, i).

Tertiary follicle (antral follicle, graff follicle); follicles containing two or more layers granulosa cells, with formed antrum follicles (Figure $1 \mathrm{j}-\mathrm{m}$ ).

\section{Measurement of serum rat $\mathrm{AMH}$}

The serum level of rat AMH was determined using a commercial enzyme-linked immunosorbent assay (ELISA) kits (rat ELISA kit, Sunred, Baoshan District, Shanghai, China). The rat AMH ELISA kit sensitivity was $0.101 \mathrm{ng} / \mathrm{mL}$ with a coefficient of variation of $<5 \%$. Procedures were performed according to manufacturer's instructions.

\section{Data presentation and statistics}

$\mathrm{AMH}$ levels and ovarian count evaluation was performed using the Mann-Whitney U test. Data are presented as mean \pm standard error mean, $\mathrm{p}$ values below 0.05 were considered significant. Follicle counts are also represented as percent changes.

\section{Results}

The control groups' number of primordial follicles number was 41.86 \pm 0.96 , primary follicles: $12.29 \pm 0.42$, secondary follicles: $9.00 \pm 0.66$, and tertiary follicles: $23.29 \pm 0.81$ (Table 1, Figure 1).

At the end of four weeks of low-dose $H$. perforatum treatment (100 $\mathrm{mg} / \mathrm{kg}$ ), the primordial follicles number increased by $2.03 \%(42.71 \pm 1.11)$, but decreased by $11.63 \%(10.86 \pm 0.55)$, $31.7 \%(6.14 \pm 0.40)(\mathrm{p}<0.01)$, and 62.6\% (8.71 \pm 0.61$)(\mathrm{p}<0.01)$ for primary, secondary, and tertiary follicles, respectively. Four weeks after ceasing $H$. perforatum treatment, the primordial follicle number $(44.86 \pm 1.28)$ was still $7.1 \%$, slightly higher than the control $(41.86 \pm 0.96)$, the primary follicle number was nearly equal to the control value $(12.43 \pm 0.37)$, the secondary follicle number was $65.1 \%$, which was higher than the control $(14.86 \pm 0.60)(p<0.01)$, and tertiary follicles had recovered 

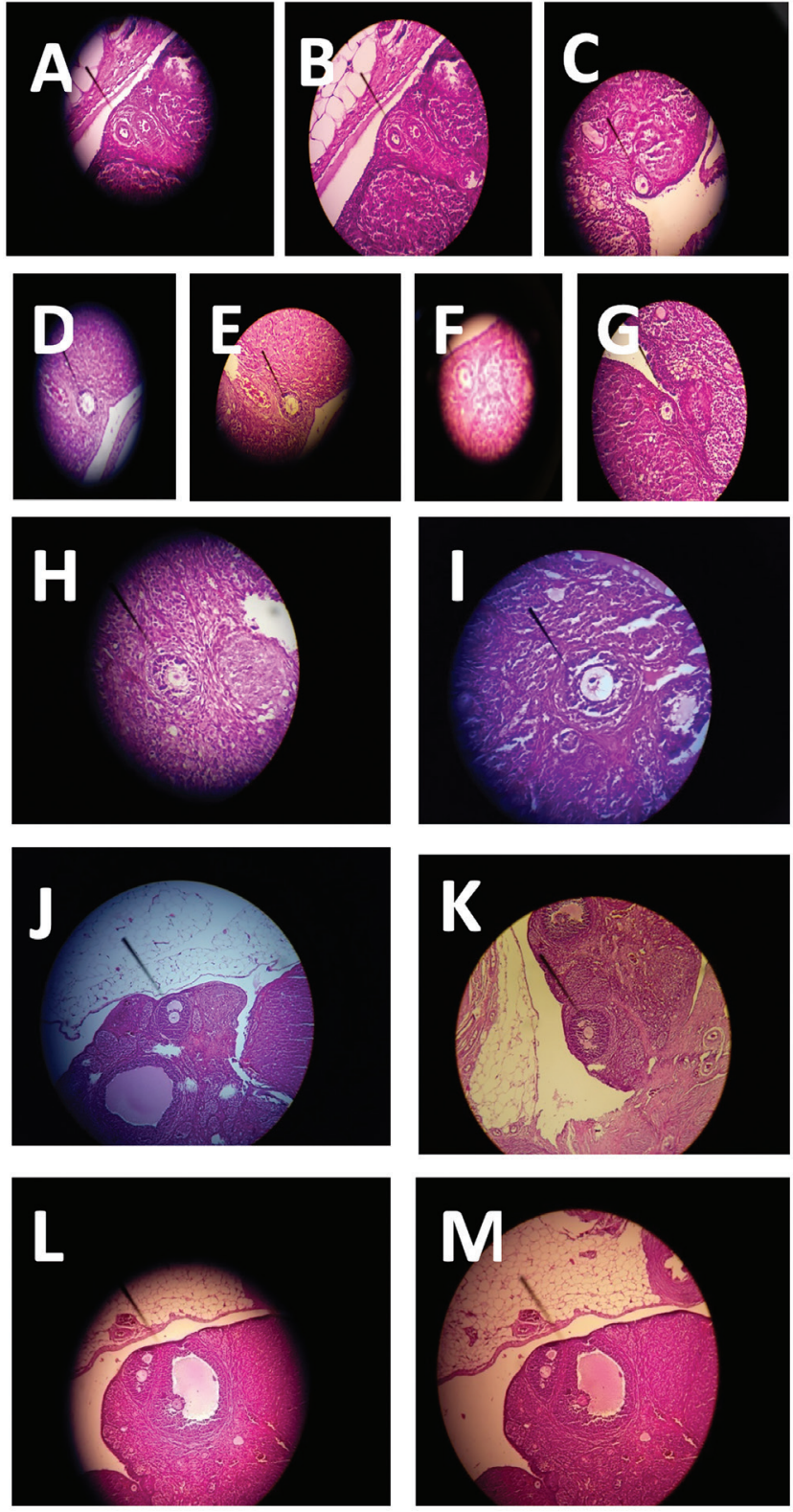

Figure 1a-m. Pictures of primordial (a, b, c magnification $\times 10)$ and primer follicles $(d, e, f \times 10$ magnification; $g$ Magnification $\times 40)$, pictures of secondary ( $h$, i magnification $\times 10)$ and tertiary follicles $(j, k \times 10$ magnification; $1, \mathrm{~m}$ magnification $\times 40$ ) mostly, but still remained 10.4\% $(20.86 \pm 0.96)(\mathrm{p}<0.05)$ lower than the control value.

In the high-dose $H$. perforatum treatment group ( $300 \mathrm{mg} / \mathrm{kg})$, the primordial follicle count dropped 29.0\% (29.71 1.15$)(\mathrm{p}<0.01)$, and after the waiting period of four weeks the number was even lower $43.6 \%(23.57 \pm 0.84)(\mathrm{p}<0.01)$. Although the primary follicle number did not change in both situations $(11.57 \pm 0.65$, $11.71 \pm 0.92)$, the number of secondary follicles did not change at the end of treatment $(8.86 \pm 0.51)$, but declined $65.1 \%(3.14 \pm 0.60)$ $(p<0.01)$ four weeks later. At the end of treatment, the decline of tertiary follicles was $87.7 \%(2.86 \pm 0.74)(\mathrm{p}<0.01)$, and recovered to $9.29 \pm 0.68$ but remained $60.1 \%(\mathrm{p}<0.01)$ lower than control.

Serum AMH level of the three groups were not significantly different from each other ( $p>0.05)$; the control group's $\mathrm{AMH}$ level was found as $4.39 \pm 0.47 \mathrm{ng} / \mathrm{mL}$, the low-dose $H$. perforatum group was $4.63 \pm 0.63 \mathrm{ng} / \mathrm{mL}$, and the high-dose $H$. perforatum group was $5.02 \pm 0.29 \mathrm{ng} / \mathrm{mL}$.

\section{Discussion}

Many researchers have focused on the beneficial anti-depressive effects of $H$. perforatum, and despite there being few reports about its harmful effects, unfortunately, this does not signify the plant's safety, it shows a lack of rigorous research. This over-thecounter plant is available in many countries as an alternative treatment modality to anti-depressive medicines (5). The EMA determined in its guidelines the adult dosage is a total $900 \mathrm{mg} /$ day (three-times $300 \mathrm{mg} /$ day) for the treatment of depression (5). Unfortunately, it has been reported that it can be abused among adolescents who believe it can make them feel good (8), in this case, its limited upper dose has not been established due to its non-prescription use. Depression can affect people of any age and disturb fertility, and any woman could be exposed to $H$. perforatum, assuming that it is safe, either before being pregnant and even while pregnant. Therefore, this study has investigated harmful effects of $H$. perforatum on ovarian tissue in two different doses and evaluated reversibility and if there was any alteration on ovarian reserve.

In this study, $100 \mathrm{mg} / \mathrm{kg}$ doses of $H$. perforatum did not inhibit the primordial stage, but when the dose was tripled, these parts

Table 1. Ovarian follicles counting of all experimental groups

\begin{tabular}{|l|l|l|l|l|}
\hline & $\begin{array}{l}\text { Primordial } \\
\text { follicles }\end{array}$ & $\begin{array}{l}\text { Primary } \\
\text { follicles }\end{array}$ & $\begin{array}{l}\text { Secondary } \\
\text { follicles }\end{array}$ & $\begin{array}{l}\text { Tertiary } \\
\text { follicles }\end{array}$ \\
\hline Control & $41.86 \pm 0.96$ & $12.29 \pm 0.42$ & $9.00 \pm 0.66$ & $23.29 \pm 0.81$ \\
\hline $100 \mathrm{mg} / \mathrm{kg}$ H. perforatum & $42.71 \pm 1.11$ & $10.86 \pm 0.55$ & $6.14 \pm 0.40 \#$ & $8.71 \pm 0.61 \#$ \\
\hline $100 \mathrm{mg} / \mathrm{kg}$ H. perforatum + waiting period & $44.86 \pm 1.28$ & $12.43 \pm 0.37$ & $14.86 \pm 0.60 \#$ & $20.86 \pm 0.96 *$ \\
\hline $300 \mathrm{mg} / \mathrm{kg}$ H. perforatum & $29.71 \pm 1.15 \#$ & $11.57 \pm 0.65$ & $8.86 \pm 0.51$ & $2.86 \pm 0.74 \#$ \\
\hline $300 \mathrm{mg} / \mathrm{kg}$ H. perforatum + waiting period & $23.57 \pm 0.84 \#$ & $11.71 \pm 0.92$ & $3.14 \pm 0.60 \#$ & $9.29 \pm 0.68 \#$ \\
\hline $\mathrm{n}=7 ;{ }^{*} \mathrm{p}<0.05 ;$ \# $<0.01$ & & & & \\
\hline
\end{tabular}


of the follicles were also affected. The primary follicle number remained the same in both doses; secondary and tertiary follicles numbers decreased in a dose-dependent manner and the ovarian cell count waiting did not recover after a four-week treatment cessation period. An earlier study showed that onehour pretreatment of $H$. perforatum $(0.6 \mathrm{mg} / \mathrm{mL})$ resulted in zero penetration of hamster zona-free oocytes and significant denaturation of sperm DNA to decrease the sperm viability, leading to concerns that its use may lead to decreased fertility (9). When $H$. perforatum was given as $180 \mathrm{mg} / \mathrm{kg}$ to mice two weeks before mating, the authors found no specific concerns about the pregnancy rates of the treated and non-treated groups; however, the study was focused on pup development and the number of live pups per litter were not found different between the groups (3).

There are no toxicity reports with regard to exposure to $H$. perforatum on direct ovarian tissues in the literature, but it is possible to find some deleterious effects on other organs. Gregoretti et al. (10) gavaged rats during gestation and for 21 days during lactation at doses 100 or $1000 \mathrm{mg} / \mathrm{kg}$ and renal and hepatic damage was identified in the pups at both doses. We did not want to use $1000 \mathrm{mg} / \mathrm{kg}$ doses of $H$. perforatum, which seem very high, our aim was just to mimic real life and worked with lower-moderate doses as $100 \mathrm{mg} / \mathrm{kg}$ and $300 \mathrm{mg} /$ $\mathrm{kg}$, following Rayburn et al. (3) study.

We also searched reports of HP on hormonal status in the literature. One study evaluated $H$. perforatum (900 mg/kg) interaction with oral contraceptive therapy in sixteen healthy women. As the CPY3A4 enzyme was induced, which is wellknown aspect of $H$. perforatum, the metabolism of these hormonal components was increased by approximately $25 \%$ and resulted in breakthrough bleeding, pre-ovulatory follicles and follicle exceeding (11). Thirty-six women aged 18-45 years with regular menstrual cycles who were diagnosed as having mild premenstrual syndrome were given $H$. perforatum (900 $\mathrm{mg} / \mathrm{day}$ ); in the follow-up, there were no differences in plasma follicle-stimulating hormone, luteinizing hormone, estradiol, progesterone, prolactin, and testosterone found compared with the non-treated group (4). Contrarily, hypo-prolactinemic activity has been reported in healthy male volunteers and plasma growth hormone levels increased due to dopaminergic action of $H$. perforatum (12). Plasma cortisol levels were significantly elevated in only four male volunteers out of twelve (12). Similarly, Di Carlo et al. (13) showed an inhibitory activity of $H$. perforatum $(100 \mathrm{mg} / \mathrm{kg})$ on prolactin production in male rats with 15 days' treatment and suggested its clinical reflection might be luteal inadequacy, probably observed due to following pharmacologically-induced low prolactin secretion. An animal study on the hypothalamic-pituitary-adrenal axis
(HPA) determined that two weeks' treatment with hypericin $(0.2 \mathrm{mg} / \mathrm{kg})$ significantly down-regulated circulating plasma levels of ACTH and corticosterone; the authors suggested that this flavonoid of $H$. perforatum played an important role in the modulation of HPA axis function (14). Considering that stress and anxiety activate the HPA, and this activation can disturb the hormones of fertility (1), H. perforatum treatment sounds beneficial, but this approach requires detailed analysis. These previously published reports show that $H$. perforatum has the capability to interact with endocrine pathways; at some point, this might be helpful to explain the detrimental effect of $H$. perforatum on follicle growth. Interestingly, it was not possible to find a current study about $H$. perforatum and hormone profiles.

$\mathrm{AMH}$ is produced by the granulosa cells of early developing follicles and inhibits the transition from primordial follicles to the primary follicular stage. AMH can be measured in serum and has been shown to be proportional to the number of small antral follicles (15). It has been suggested that there is a strong positive correlation between serum $\mathrm{AMH}$ levels and antral follicle count; the use of AMH combined with antral follicle counts may improve ovarian reserve evaluation (16). In our study, we found no significant changes in AMH levels and also the primary follicle reserve remained stable with both doses of H. perforatum.

It has been clearly shown that $H$. perforatum treatment decreased follicle counts in a dose-dependent manner, especially primordial and tertiary pools. This study should help navigate future research on the hormonal aspect of the effect of $H$. perforatum, which has to be investigated in detail. Any woman who takes $H$. perforatum could experience ovarian failure.

Ethics Committee Approval: Adnan Menderes University Animal Ethical Committee's Approval, HADYEK64583101/2016/3.

\section{Informed Consent: It was taken.}

Peer-review: Externally peer-reviewed.

Author Contributions: Concept - B.D., T.A.; Design - B.D.; Supervision - B.D., T.A., Y.Ö.; Materials: F.K., M.Y., Y.Ö.; Writer - B.D.

Conflict of Interest: No conflict of interest is declared by the authors.

Financial Disclosure: The authors declared that this study received no financial support. 


\section{References}

1. Mitsi C, Efthimiou K. Infertility: psychological-psychopathological consequences and cognitive-behavioural interventions. Psychiatriki 2014; 25: 293-302.

2. O'Reilly E, Sevigny M, Sabarre KA, Phillips KP. Perspectives of complementary and alternative medicine (CAM) practitioners in the support and treatment of infertility. BMC Complement Altern Med 2014; 14: 394.

3. Rayburn WF, Gonzales CL, Christensen HD, Stewart JD. Effect of prenatally administered Hypericum (Saint John's wort) on growth and physical maturation of mouse offspring. Am J Obstet Gynecol 2001; 184: 191-5.

4. Canning S, Waterman M, Orsi N, Ayres J, Simpson N, Dye L. The Efficacy of Hypericum perforatum (St John's Wort) for the Treatment of Premenstrual Syndrome: A Randomized, DoubleBlind, Placebo-Controlled Trial. CNS Drugs 2010; 24: 207-25.

5. European Medicines Agency, Evaluation of Medicines for Human Use London, 30 January 2018 EMA/HMPC/244315/2016 Committee on herbal medicinal products (HMPC). Hypericum perforatum L., herba. European Medicines Agency, 2018.

6. Pedersen T, Peters H. Proposal for a classification of oocytes and follicules in the mouse ovary. J Reprol Fertil 1968; 17: 555-7.

7. Xu C, Zhao Y. Apoptosis of rat's ovarian follicle cells induced by triptolide in vivo. African Journal of Pharmacy and Pharmacology 2010; 4: 422-30.

8. Biggs JM, Morgan JA, Lardieri AB, Kishk OA, Klein-Schwartz W. Abuse and misuse of selected dietary supplements among adolescents: A look at poison center data. J Pediatr Pharmacol Ther 2017; 22: 385-93.
9. Ondrizek RR, Chan PJ, Patton WC, King A. An alternative medicine study of herbal effects on the penetration of zona-free hamster oocytes and the integrity of sperm deoxyribonucleic acid. Fertil Steril 1999; 71: 517-22.

10. Gregoretti B, Stebel M, Candussio L, Crivellato E, Bartoli F, Decorti G. Toxicity of Hypericum perforatum (Saint John's wort) administered during pregnancy and lactation in rats. Toxicol Appl Pharmacol 2004; 200: 201-5.

11. Murphy PA, Kern SE, Stanczyk FZ, Westhoff CL. Interactions of St. John's wort with oral contraceptives: effects on the pharmacokinetics of norethindrone and ethinyl estradiol, ovarian activity and breakthrough bleeding. Contraception 2005; 71: 402-8.

12. Franklin M, Chi J, McGavin C, Hockney R, Reed A, Campling G, et al. Neuroendocrine evidence for dopaminergic actions of Hypericum extract (LI 160) in healthy volunteers. Biol Psychiatry 1999; 46: 581-4.

13. Di Carlo G, Pacilio M, Capasso R, Carlo R. Effect on prolactin secretion of Echinacea purpura, Hypericum perforatum and Eleutherococcus senticosus. Phytomedicine 2005; 12: 644-7.

14. Butterweck V, Hegger M, Winterhoff H. Flavonoids of St. John's Wort reduce HPA axis function in the rat. Planta Med 2004; 70: 1008-11.

15. La Marca A, Broekmans FJ, Volpe A, Fauser BC, Macklon NS; ESHRE Special Interest Group for Reproductive Endocrinology AMH Round Table. Anti-Mullerian hormone (AMH): what do we still need to know? Human Reproduction 2009; 24: 2264-75.

16. Barbakadze L, Kristesashvili J, Khonelidze N, Tsagareishvili G. The correlations of anti-mullerian hormone, follicle-stimulating hormone and antral follicle count in different age groups of infertile women. Int J Fertil Steril 2015; 8: 393-8. 